\title{
X-RAY MAGNETIC SCATTERING
}

\author{
M.J. COOPER \\ Department of Physics, University of Warwick, Coventry CV4 7AL, UK
}

\begin{abstract}
Magnetic X-ray diffraction with synchrotron radiation is now an established technique for studies of antiferromagnets. The problems associated with the small magnetic scattering cross-section being alleviated by the dramatic enhancements found near absorption edges through resonant exchange scattering. The technique is particularly useful for those materials that require high wavevector resolution to reveal the structural phase transitions that accompany the magnetic ordering process or those that are difficult to investigate with neutrons, (e.g. samarium, for which recent results are presented). In the actinides the work is also motivated by the objective of performing an empirical separation of the spin and orbital components of magnetisation. Diffraction studies of ferromagnets require circular polarised radiation and suffer from the superposition of the small magnetic signal and the charge scattering; to date Laure methods have proved more successful than monochromatic beam studies. Ferro- and ferrimagnets can also be studied by Compton (inelastic) scaltering but the cross-section is less well established: considerable effort has been directed to determining whether orbital magnetisation can be measured in these experiments and results on $\mathrm{HoFe}_{2}$ now indicate this is not so. Magnetic Compton profiles provide information about the momentum distribution of electrons with unpaired spins, and this, together with magnetisation data can provide the basis for the separation of spin and orbital magnetisation.
\end{abstract}

PACS numbers: 61.10.Dp, 75.50.-y, 78.70.-g, 78.70.Ck

\section{Introduction}

Magnetic X-ray scattering is no longer the novelty that it was when de Bergevin and Brunel first demonstrated magnetic diffraction effects with a commercial X-ray tube sources [1] and Sakai and Ono performed a magnetic Compton scattering experiment with gamma rays emitted by a feeble radioisotope [2] in the nineteen seventies. "Exploitation" is beginning to follow "demonstration" as synchrotron sources of high brightness X-rays become available and as the theory is put on a firm footing. Seminal steps in the past decade include the elaboration of the theory of magnetic diffraction for various geometries and photon polarisations [3-5] and the observation (plus subsequent explanation) of resonance exchange scattering at absorption edges [6-8]. It is, of course too early to identify the landmarks of this decade, but they will surely be associated with the new generation 
of synchrotrons shortly to come on stream in three continents (ESRF in Europe, APS in North America and SPring-8 in Japan). These sources should not only facilitate the exploitation of those magnetic scattering effects whose potential is already known but also present us with new phenomena to understand.

Several properties of synchrotron radiation are vital for the prosecution of magnetic scattering studies. The first is the brightness, i.e. the flux of radiation within a stated angular aperture; the high resolution in $\boldsymbol{K}$ space needed to separate charge and magnetic Bragg peaks in complicated structures implies the use of highly collimated radiation. The next generation of synchrotrons are machines with low "emittance" which means that the radiation is naturally highly collimated and is emitted from a very small source. The latter property makes the source not only "bright" but "brilliant" and means that if focussing optics are used, at the expense of some loss in resolution, very small samples can be studied. The final property associated with the low emittance of these sources is the high degree of polarisation (0.995) of the radiation; this is important in some techniques of separating charge from magnetic scattering. Consider scattering at $90^{\circ}$ in the orbital plane of the synchrotron. The radiation is plane polarised in that plane and if, for example, $P_{\mathrm{L}}=0.995$, the charge scattering (amplitude $\propto 1-P_{\mathrm{L}}$ ) would be almost entirely supressed, the magnetic scattering on the other hand involves polarisation flipping and such a signal would still occur. The ability to choose X-ray energies such that Bragg angles of $45^{\circ}$ can be contrived is another asset of the synchrotron source; it has a continuous spectrum from which monochromatic beams of different energies can be selected.

\section{The theory of magnetic scattering}

There are now sufficient papers in the literature to excuse the briefest of summaries being given here. Scattering is a second order process and so, in terms of the semi-classical Hamiltonian $(p+A e / c)^{2} / 2 m$, contributions to the scattering arise from the $\boldsymbol{A}^{2}$ term in the first order and the $\boldsymbol{p} \cdot \boldsymbol{A}$ term in the second order. The former being responsible for ordinary scattering (the Thomson term) and the latter for the anomalous dispersion contributions. Relativistic corrections can then be introduced in a perturbation approach which attaches a term of the form $\boldsymbol{s} \cdot(\boldsymbol{A} \times \boldsymbol{A})$ to the Thomson scattering and one in $s \cdot \operatorname{curl} A$ to the dispersion corrections. The perturbation expansion appears in amplitude terms of order $g=\left(E_{x} / m c^{2}\right)$. This method of calculation by approximation is essential for electrons bound in atomic potentials as is pointed out by Balcar and Lovesey [9]. The general expressions for magnetic scattering to order $g$, which is adequate for elastic scattering and to order $g^{2}$ which is necessary for inelastic scattering and for higher photon energies can be found in the paper by Grotch et al. [10]. Rather simpler manifestations of the same cross-section terms can be found in the papers of Blume [3], Lovesey $[4,11]$. 
The general form of the scattering amplitude, to the first order in $g$, can be written as follows:

$$
\begin{aligned}
& \frac{\mathrm{d} \sigma}{\mathrm{d} \Omega}=\left(\frac{e^{2}}{m c^{2}}\right)^{2}\left\{\left\langle i\left|\sum_{j} \exp \left(i \boldsymbol{K} \cdot \boldsymbol{r}_{j}\right)\right| i\right\rangle \boldsymbol{A}\right. \\
& -\frac{i E_{x}}{m c^{2}}\left[\left\langle i\left|\sum_{j} \exp \left(i \boldsymbol{K} \cdot \boldsymbol{r}_{j}\right) \boldsymbol{S}_{j} \cdot \boldsymbol{B}\right| i\right\rangle\right. \\
& \left.+\left\langle i\left|\sum_{j} \exp \left(i \boldsymbol{K} \cdot \boldsymbol{r}_{j}\right) \frac{\boldsymbol{K} \cdot \boldsymbol{p}}{\hbar^{2} \boldsymbol{K}^{2}} \cdot C\right| i\right\rangle\right\}^{2},
\end{aligned}
$$

where $\boldsymbol{A}, \boldsymbol{B}$, and $\boldsymbol{C}$ are polarisation factors and $\boldsymbol{K}$ is the $\mathrm{X}$-ray scattering vector and the sum is over the $j$ target electrons. The first magnetic term is clearly associated with spin whilst the second is the orbital operator. Several observations can be made immediately. Firstly the pre-factor $g$ associated with all the magnetic amplitudes indicates that the intensity of magnetic scattering will be low at conventional X-ray energies, especially when it is remembered that all the electrons contribute to the first (Thomson) term whereas only those with unpaired spin or orbital moments contribute to the magnetic scattering. Secondly the magnetic scattering is in quadrature with the charge scattering so that pure magnetic scattering occurs with a relative intensity of $g^{2}$. (This was the term responsible for the observed scattering in the first experiments with the antiferromagnet $\mathrm{NiO}$; even though the magnetic superlattice peaks appear away from the charge scattering their observation was a veritable tour de force.) Thirdly as a positive point in contrast to these two negative ones, the existence of different polarisation factors in the spin and orbital terms means that, in contrast to the situation encountered in magnetic neutron scattering, there is a possibility of separating spin and orbital magnetisation.

First order magnetic scattering (intensity $\propto g$ ) is actually present under many experimental conditions; for example when the form factor is complex, as arises if the structure is non-centrosymmetric or if the experiment is carried out at a photon energy near an absorption edge of the sample (anomalous dispersion). In both cases there will be a real overlap term in the cross-section when unpolarised or linearly polarised radiation is used. Another method of producing a real interference term between the charge and magnetic scattering arises when the photon polarisation (vectors $B$ and $C$ ) are complex, i.e. when circular polarisation is used. This can be engineered with synchrotron radiation as, of course, can the selection of photon energies tuned to absorption edges. In fact this latter procedure has led to one of the most exciting discoveries of magnetic scattering so far: resonant exchange scattering.

\section{Resonant exchange $X$-ray scattering}

The observation [6] that the intensity of the $c$-axis magnetic satellites in Ho (a spiral antiferromagnet) increased by a factor of approximately 50-fold when 


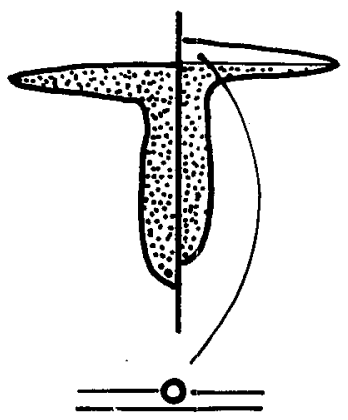

Fig. 1. Schematic diagram illustrating the origin of resonant exchange scattering.The exchange splitting leads to a high density of unoccupied states in one of the sub-bands. The levels are further narrowed by the presence of the core hole which increases the binding of the excited level.
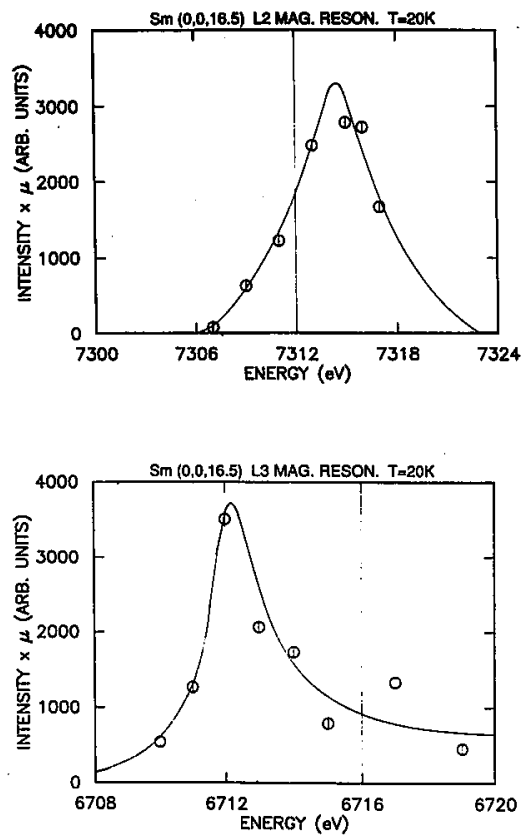

Fig. 2. Energy scans of the $(0,0,16.5)$ magnetic satellite reflection through the $L_{1 \mathrm{II}}$ and $L_{\text {III }}$ resonances in samarium. The vertical line indicated the position of the absorption edges; the curves are merely guides to the eye. Data are taken from [12].

the X-ray energy was tuned through the $L_{\mathrm{III}}$ absorption edge was explained [7] in terms of resonant electric quadrupole transitions between $2 p$ to $4 f$ levels together with dipole transitions between $2 p$ to $5 d$ levels. These, by themselves are not inherently "magnetic" but in actual fact the final states only exist as unoccupied 
states because they are emptied by the magnetic exchange splitting as shown schematically in Fig. 1.

The enhancement factor is high when the density of unoccupied states is high, i.e. with tightly bound atomic-like states. The presence of the core hole increases the binding of the upper state and thereby contributes to the enhancement. Figure 2 shows results obtained at the Daresbury Storage Ring Source on samarium [12] for the $L_{\mathrm{II}}$ and $L_{\mathrm{III}}$ resonances, which peak at opposite sides of the absorption edges.

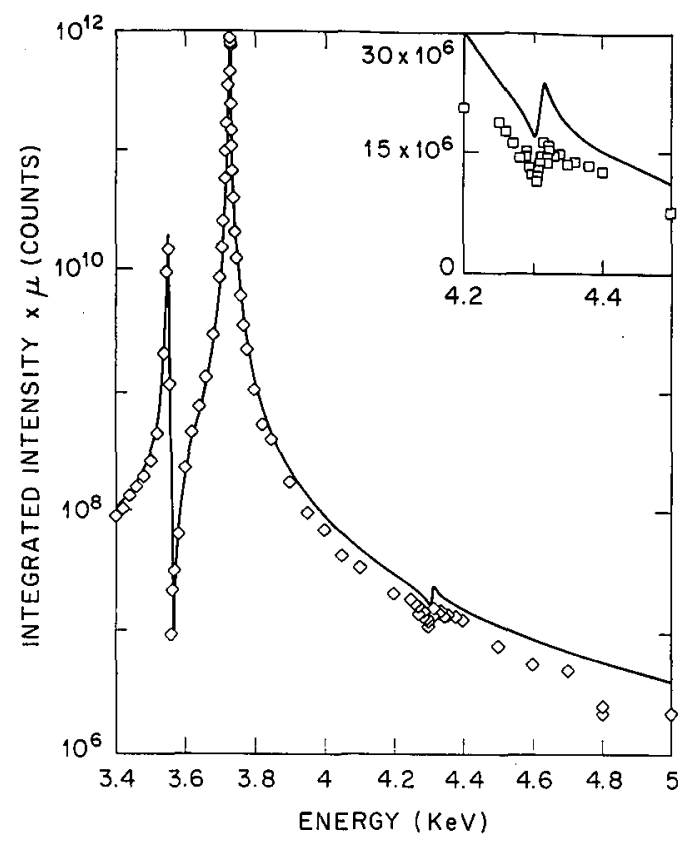

Fig. 3. Energy variation of the $(0,0,5.5)$ reflection in UAs through the $M_{\mathrm{V}}, M_{\mathrm{IV}}$ and (inset) $M_{\text {III }}$ edges. Data reproduced from [13].

The asymmetric line shapes observed in both cases may be attributable to interference between resonant and non-resonant contributions. Samarium is an interesting example of the potential for X-ray magnetic scattering to complement neutron studies. Unless isotopically-enriched material is used it is very difficult to study with neutrons; it also has a very small moment $\left(0.1 \mu_{\mathrm{B}}\right)$ and an unusual form factor (it does not peak at the origin) due to the opposition of spin and orbital moments.

The enhancement factor depends on the density of final states uncovered by the exchange splitting and the factor of 50 for Ho pales into insignificance compared with factors of $10^{6}$ observed in studies of UAs [13] (see Fig. 3), other actinides and transuranium compounds where there is a high density of atom-like $5 d$ states. The existence of such resonant effects means that magnetic scattering signals that might otherwise be considered too weak to be measurable are at resonance $>0.1 \%$ 
of the charge scattering and therefore easily distinguished. Another aspect of the resonant exchange scattering is that the branching ratios that are measured are very sensitive to crystal field splittings. Thus not only can the valence state of the ion be deduced, but calculated crystal field effects can be tested. Polarisation analysis of the scattered beam (given that the incident beam has a well-defined linear polarisation in the plane of the synchrotron) allows the quadrupolar and dipolar contributions to the resonance to be separated, providing yet another test of the theory. This requires higher synchrotron flux brightness than is yet available at many storage rings. So far the measurements have been limited to antiferromagnets although significant enhancements have been predicted for ferromagnetic materials such as $\mathrm{MnPt}_{3}[8]$.

\section{Diffraction studies of ferromagnets}

The problem of measuring magnetic diffraction peaks when they are superimposed on the charge scattering has proved to be very difficult to date and has only been sucessfully tackled by the use of white rather than monochromatised beam techniques pioneered at Daresbury Laboratory by Collins et al. [14]. Circu-

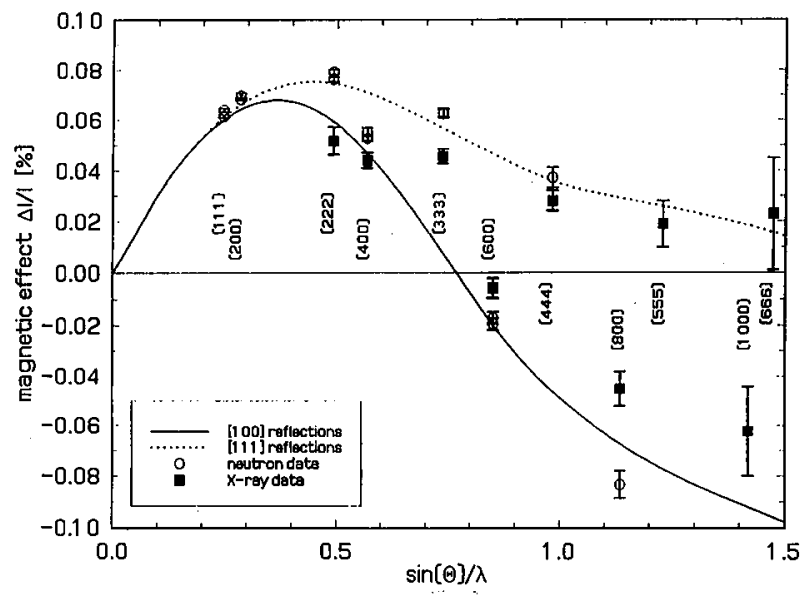

Fig. 4. Magnetic diffraction observed in $\mathrm{Ni}$ using the white beam technique. The results show clearly both the anisotropy in the magnetic form factor and the weighting that the method gives to high order magnetic reflections. (Zukowski et al. private comamenication).

larly polarised photons must be used to obtain a first order term (second order contributions would be immeasureably small). They show, in a study of $\mathrm{Fe}$, that the pre-factor $g=E_{x} / m c^{2}$ means that the higher order magnetic reflections, which occur at higher photon energies, can be measured with better precision than is possible in neutron studies; by contrast the reverse appears to be true, at least at the present level of precision, with the low energy, low index reflections. A typical data set, obtained by the author's group (Zukowski et al., private communication) is shown in Fig. 4. 


\section{Inelastic (Compton) scattering}

Historically speaking magnetic Compton scattering preceded diffraction in its observation. A Compton polarimeter was used to measure the degree of circular polarisation of gamma rays emitted by cooled oriented nuclei as early as 1955 [15]. The cross-section for magnetic Compton scattering was first calculated by several authors for a free, stationary spin-polarised electron [16]. The impulse approximation was then invoked to validate the use of this formula to describe the scattering from a bound electron with spin, i.e. one moving in a potential. In this approach [17] the question of contributions from orbital scattering did not occur. This, together with the fact that Compton scattering experiments are carried out under conditions of backscattering that would in any event supress any orbital scattering, has led to considerable current interest into the question of whether the orbital term is present in incoherent scattering experiments carried out in the regime of the impulse approximation which essentially requires the energy transfer to the target electron to be sufficient to eject it into a plane wave continuum state. Whilst the theory of Grotch et al. [10] has been extended to include this case by Lovesey [18] others have queried whether the measurement of an orbital component is compatible with the instantaneous nature of the interaction implied by the impulse approximation. Recent work from the author's group on this problem is presented below.

\section{Recent Compton scattering results}

According to $[18,19]$ the intensity of the magnetic Compton scattering is a function of both the angle of scattering $\theta$ and the angle $\alpha$ between the direction of the magnetic field and the incident wavevector. It can be written as follows:

$$
\left(E / m c^{2}\right) P_{c}\left[F_{s} \xi(\theta, \alpha)+F_{L} \zeta(\theta, \alpha)\right]
$$

where

$$
\begin{aligned}
\xi(\theta, \alpha)= & (1-\cos \theta)(2 \cos \theta \cos \alpha+\sin \theta \sin \alpha)+ \\
& +(\varepsilon / 2)[\cos \alpha(1+3 \cos \theta)(1-\cos \theta)+\sin \theta \sin \alpha(1-3 \cos \theta)]
\end{aligned}
$$

and

$$
\begin{aligned}
\zeta(\theta, \alpha)= & {\left[\frac{\sin \theta(1+\cos \theta)}{2(2+\varepsilon)}\right] } \\
& \times\left\{4 \sin \frac{\theta}{2} \cos (\alpha-\theta / 2)+\varepsilon[\sin \alpha+(3+\varepsilon) \sin (\theta-\alpha)]\right\} .
\end{aligned}
$$

$P_{\mathrm{C}}$ is the degree of circular polarisation and $\varepsilon$ is the fractional energy change, which is 0.086 in the experiments discussed below. $F_{S}$ and $F_{L}$ are the magnetisations per unit cell originating from spin and orbital moments. Previous attempts to test this model have concentrated on determining the geometrical condition under which the two terms cancel each other for the soft ferromagnets $\mathrm{Fe}$ and Co dominated by spin contributions [19]. A new experiment has now been performed at the Accumulation ring in Japan by a joint UK/Japanese team, aimed at providing a 

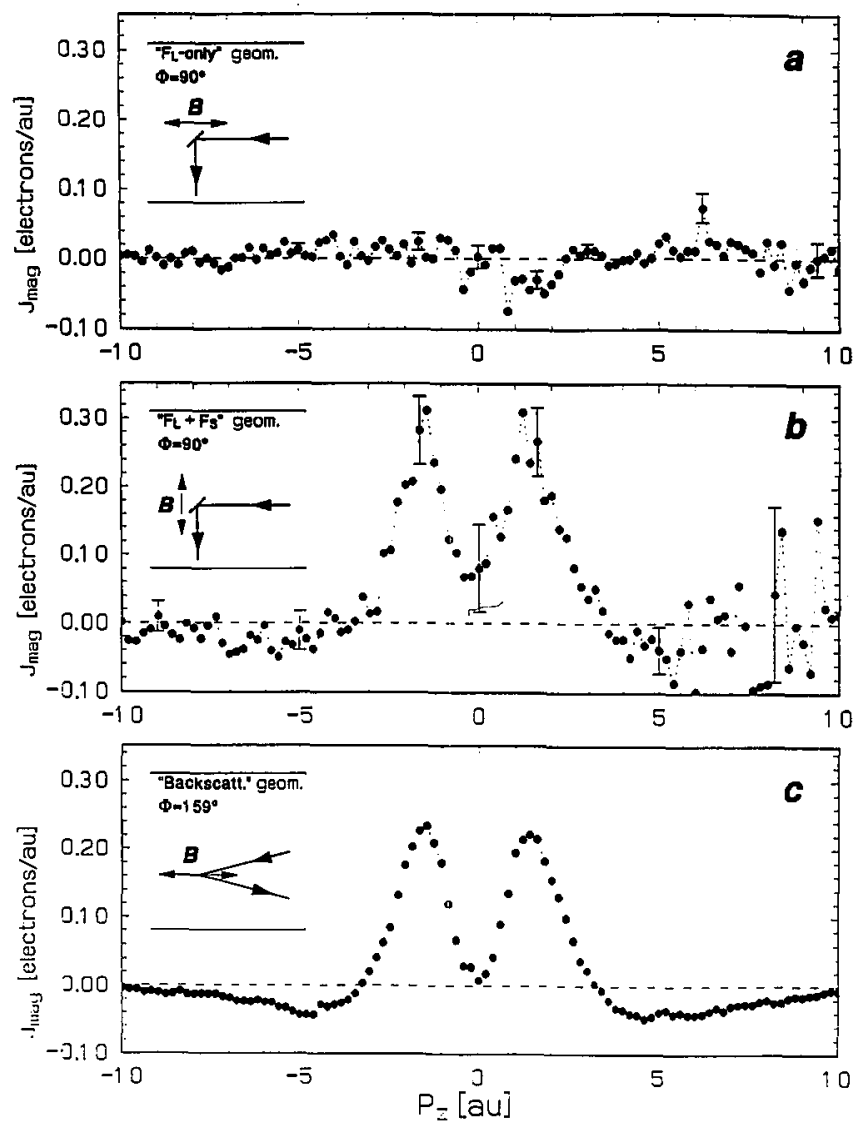

Fig. 5. Magnetic Compton scattering from $\mathrm{HoFe}_{2}$ under different geometrical conditions; the spectra are plotted on a scale of electron momenta ( 1 au $=1.99 \times$ $10^{-24} \mathrm{~kg} \mathrm{~m} \mathrm{~s}{ }^{-2}$ ). The upper diagram shows that no significant scattering occurs in the geometry that was thought to favour orbital scattering whereas the middler diagram shows a profile which is clearly close to that obtained in backscattering when spin-only contributions are important. Data taken from [20].

more incisive test of the theory [20]. The expression in Eq. (2) takes on a much simpler form when the scattering angle is $90^{\circ}$, viz.:

$$
F_{S}\left[\sin \alpha+\frac{1}{2} \varepsilon(\cos \alpha+\sin \alpha)\right]+\frac{1}{2} F_{L}[\sin \alpha+(1+\varepsilon) \cos \alpha]
$$

and in particular when $\alpha=0^{\circ}$ (field parallel to the incident beam) the expression is

$$
\propto\left[F_{S} \varepsilon / 2+F_{L}(1+\varepsilon) / 2\right]
$$

and for $\alpha=90^{\circ}$ (magnetic field parallel to the scattered beam)

$$
\propto\left[F_{S}(1+\varepsilon / 2)+F_{L} / 2\right] .
$$


This magnetic contribution can be isolated by taking the difference between spectra with the magnetic field alternately parallel/antiparallel to the chosen direction. In the former case the scattered intensity should be almost entirely from the orbital term whereas in the latter both contributions should be significant. The experiment was carried out on $\mathrm{HoFe}_{2}$ the magnetisation of which is dominated by the orbital moment on Ho which is opposed by the spin moment associated with the Fe sites $\left(F_{L} / F_{S}=-3\right.$ at room temperature). If orbital magnetisation was present in this material the upper spectrum in Fig. 5 should have a peak with a similar height to that in the lower figure, but with a characteristically different shape - single peaked and broader. In fact nothing of statistical significance is present. However in the middle figure the magnetic Compton scattering from the same polycrystalline sample in the same magnetic field is unmistakable. Indeed, comparison with data from backscattering studies (lower diagram), which according to both spin and bound electron theory yield the spin-dependent profile alone, show that it is identical. There does not appear to be any orbital contribution to inelastic magnetic scattering in the Compton limit. This conclusion is supported by data from other synchrotron experiments by the author's group which will be published elsewhere. These results do not rule out the possiblility that there are contributions from orbital magnetisation to inelastic scattering, but they must clearly be much smaller than predicted for experiments carried out in the Compton limit.

\section{Acknowledgements}

I am grateful to the organisers of the International School on Synchrotron Radiation in the Natural Sciences, Jaszowiec, Poland, for inviting me to present this paper. The magnetic scattering studies by the Warwick group described above are supported by the UK Science and Engineering Research Council, the Japanese office of the British Council and the EEC Science Programme: I am extremely grateful to all of these organisalions. I also thank the authors who have alloved their work to be reproduced in the paper.

\section{References}

[1] F. de Bergevin, M. Brunel, Phys. Lett. A 39, 141 (1972).

[2] N. Sakai, K. Ono, Phys. Rev. Lett. 37, 351 (1976).

[3] M. Blume, J. Appl. Phys. 57, 3618 (1985).

[4] S.W. Lovesey, J. Phys. C. 20, 5625 (1987).

[5] M. Blume, D. Gibbs, Phys. Rev. B 37, 1779 (1989).

[6] D. Gibbs, D.R. Harshman, E.D. Isaacs, D.B. McWhan, D. Mills, C. Vettier, Phys. Rev. Lett. 61, 1241 (1988).

[7] J.P. Hannon, G.T. Trammell, M. Blume, D. Gibbs, Phys. Rev. Lett. 61, 1245 (1988).

[8] P. Carra, M. Altarelli, F. de Bergevin, Phys. Rev. B 40, 7324 (1989).

[9] E. Balcar, S.W. Lovesey, Theory of Neutron Scattering, Ch. 6, Oxford University, Oxford OUP 1989. 
[10] D. Grotch, E. Kazes, G. Bhatt, D.A. Owen, Phys. Rev. A 27, 243 (1983).

[11] S.W. Lovesey, in: Conference Series of Institute of Physics, No 101, 133, (1989).

[12] S.L. Lee, E.M. Forgan, S.J. Shaikh, C.C. Tang, G. Stirling, S. Langridge, A.J. Rollinson, M.M.R. Costa, M.J. Cooper, J.B. Forsyth, D. Fort, to be submitted to J. Phys. Condens. Matter (1992).

[13] D.B. McWhan, C. Vettier, E.D. Isaacs, G.E. Ice, D.P. Siddons, J.B. Hastings, C. Peters, O. Vogt, Phys Rev. B 42, 6007 (1990).

[14] S.P. Collins, D. Laundy, A.J. Rollinson, Philos. Mag. B 65, 37 (1992).

[15] J.C. Wheatley, W.J. Huiskamp, A.N. Diddens, M.J. Steeland, H.A. Tolhoek, Physica 21, 841 (1955).

[16] see for example L.D. Landau, E.M. Lifshitz, Quantum Electrodynamics, Course on Theoretical Physics, Vol. 4, 2nd Ed., Pergamon, Oxford 1982.

[17] P.M. Platzman, N. Tzoar, Phys. Rev. B 2, 3556 (1970).

[18] S.W. Lovesey, Phys. Scr. 44, 51 (1991).

[19] S.P. Collins, M.J. Cooper, S.W. Lovesey, D. Laundy, J. Phys. Condens. Matter 2, $6439(1990)$.

[20] M.J. Cooper, E. Zukowski, S.P. Collins, D.N. Timms, F. Itoh, H. Sakurai, submitted to J. Phys. Condens. Matter. (1992). 